\title{
Steganography and Steganalysis in Digital Multimedia: Hype or Hallelujah?
}

\author{
Anderson Rocha ${ }^{1,2} \quad$ Siome Goldenstein ${ }^{1}$
}

\begin{abstract}
In this tutorial, we introduce the basic theory behind Steganography and Steganalysis, and present some recent algorithms and developments of these fields. We show how the existing techniques used nowadays are related to Image Processing and Computer Vision, point out several trendy applications of Steganography and Steganalysis, and list a few great research opportunities just waiting to be addressed.
\end{abstract}

\section{Introduction}

De artificio sine secreti latentis suspicione scribendi! ${ }^{3}$. (David Kahn)

More than just a science, Steganography is the art of secret communication. Its purpose is to hide the presence of communication, a very different goal than Cryptography, that aims to make communication unintelligible for those that do not possess the correct access rights [1]. Applications of Steganography can include feature location (identification of subcomponents within a data set), captioning, time-stamping, and tamper-proofing (demonstration that original contents have not been altered). Unfortunately, not all applications are harmless, and there are strong indications that Steganography has been used to spread child pornography pictures on the internet $[2,3]$.

In this way, it is important to study and develop algorithms to detect the existence of hidden messages. Digital Steganalysis is the body of techniques that attempts to distinguish between non-stego or cover objects, those that do not contain a hidden message, and stegoobjects, those that contain a hidden message.

Steganography and Steganalysis have received a lot of attention around the world in the past few years. Some are interested in securing their communications through hiding the very own fact that they are exchanging information. On the other hand, others are interested in detecting the existence of these communications - possibly because they might be related to illegal activities.

\footnotetext{
${ }^{1}$ Institute of Computing, University of Campinas (Unicamp).

${ }^{2}$ Corresponding author: anderson.rocha@ic.unicamp.br

${ }^{3}$ The effort of secret communication without raising suspicions.
} 
In this tutorial, we introduce the basic theory behind Steganography and Steganalysis, and present some recent algorithms and developments of these fields. We show how the existing techniques used nowadays are related to Image Processing and Computer Vision, point out several trendy applications of Steganography and Steganalysis, and list a few great research opportunities just waiting to be addressed.

The remainder of this tutorial is organized as follows. In Section 2, we introduce the main concepts of Steganography and Steganalysis. Then, we present historical remarks and social impacts in Sections 3 and 4, respectively. In Section 5, we discuss information hiding for scientific and commercial applications. In Sections 6 and 7, we point out the main techniques of Steganography and Steganalysis. In Section 8, we present common-available information hiding tools and software. Finally, in Sections 9 and 10, we point out open research topics and conclusions.

\section{Terminology}

According to the general model of Information Hiding: embedded data is the message we want to send secretly. Often, we hide the embedded data in an innocuous medium, called cover message. There are many kinds of cover messages such as cover text, when we use text to hide a message; or cover image, when we use an image to hide a message. The embedding process produces a stego object which contains the hidden message. We can use a stego key to control the embedding process, so we can also restrict detection and/or recovery of the embedded data to other parties with the appropriate permissions to access this data.

Figure 1 shows the process of hiding a message in an image. First we choose the data we want to hide. Further, we use a selected key to hide the message in a previously selected cover image which produces the stego image.

When designing information hiding techniques, we have to consider three competing aspects: capacity, security, and robustness [4]. Capacity refers to the amount of information we can embed in a cover object. Security relates to an eavesdropper's inability to detect the hidden information. Robustness refers to the amount of modification the stego-object can withstand before an adversary can destroy the information [4]. Steganography strives for high security and capacity. Hence, a successful attack to the Steganography consists of the detection of the hidden content. On the other hand, in some applications, such as watermarking, there is the additional requirement of robustness. In these cases, a successful attack consists in the detection and removal of the copyright marking.

Figure 2 presents the Information Hiding hierarchy [5]. Covert channels consist of the use of a secret and secure channel for communication purposes (e.g., military covert channels). Steganography is the art, and science, of hiding the information to avoid its detection. 


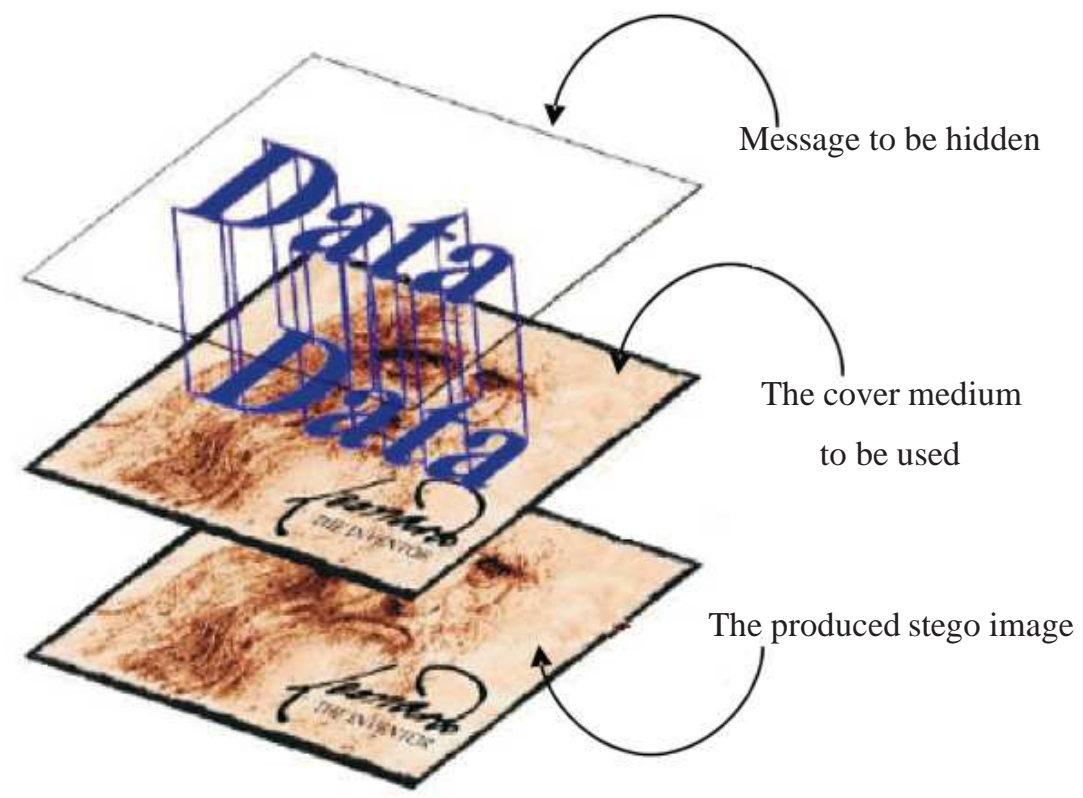

Figure 1. A data hiding example.

It derives from the Greek steganos "hide, embed" and graph "writing".

We classify Steganography as technical and linguistic. When we use physical means to conceal the information, such as invisible inks or micro-dots, we are using technical Steganography. On the other hand, if we use only "linguistic" properties of the cover object, such as changes in image pixels or letter positions, in a cover text we are using linguistic Steganography.

Copyright marking refers to the group of techniques devised to identify the ownership of intellectual property over information. It can be fragile, when any modification on the media leads to the loss of the marking; or robust, when the marking is robust to some destructive attacks.

Robust copyright marking can be of two types: fingerprinting and watermarking. Fingerprinting hides an unique identifier of the customer who originally acquired the information, recording in the media its ownership. If the copyright owner finds the document in the possession of an unwanted party, she can use the fingerprint information to identify, and prosecute, the customer who violated the license agreement. 


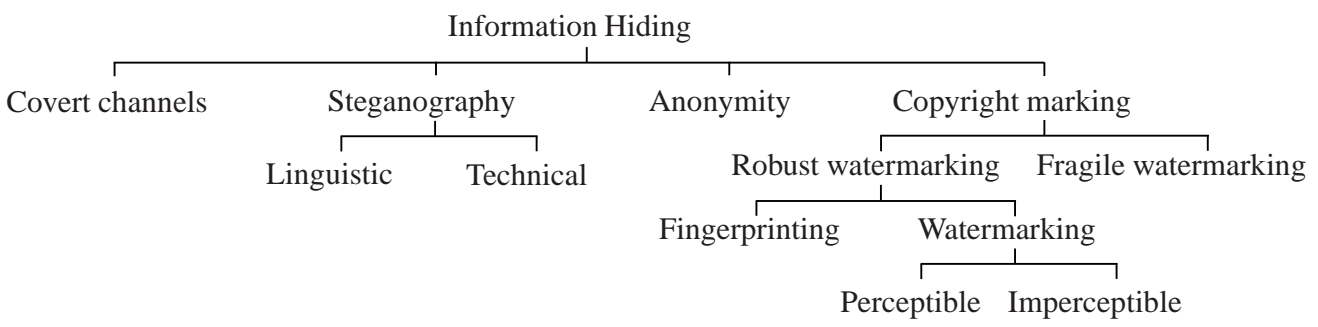

Figure 2. Information Hiding hierarchy.

Unlike fingerprints, watermarks identify the copyright owner of the document, not the identity of the owner. Furthermore, we can classify watermarking according to its visibility to the naked eye as perceptible or imperceptible.

In short, fingerprints are used to identify violators of the license agreement, while watermarks help with prosecuting those who have an illegal copy of a digital document $[5,6]$.

Anonymity is the body of techniques devised to surf the Web secretly. This is done using sites like Anonymizer ${ }^{4}$ or remailers (blind e-mailing services).

\section{Historical remarks}

Throughout history, people always have aspired to more privacy and security for their communications $[7,8]$. One of the first documents describing Steganography comes from Histories by Herodotus, the Father of History. In this work, Herodotus gives us several cases of such activities. A man named Harpagus killed a hare and hid a message in its belly. Then, he sent the hare with a messenger who pretended to be a hunter [7].

In order to convince his allies that it was time to begin a revolt against Medes and the Persians, Histaieus shaved the head of his most trusted slave, tattooed the message on his head and waited until his hair grew back. After that, he sent him along with the instruction to shave his head only in the presence of his allies.

Another technique was the use of tablets covered by wax, first used by Demeratus, a Greek who wanted to report from the Persian court back to his friends in Greece that Xerxes, the Great, was about to invade them. The normal use of wax tablets consisted in writing the text in the wax over the wood. Demeratus, however, decided to melt the wax, write the message directly to the wood, and then put a new layer of wax on the wood in such a way

${ }^{4}$ www . anonymizer. com 
that the message was not visible anymore. With this ingenious action, the tablets were sent as apparently blank tablets to Greece. This worked for a while, until a woman named Gorgo guessed that maybe the wax was hiding something. She removed the wax and became the first woman cryptanalyst in History.

During the Renaissance, the Harpagus' hare technique was "improved" by Giovanni Porta, one of the greatest cryptologists of his time, who proposed feeding a message to a dog and then killing the dog [8].

Drawings were also used to conceal information. It is a simple matter to hide information by varying the length of a line, shadings, or other elements of the picture. Nowadays, we have proof that great artists, such as Leonardo Da Vinci, Michelangelo, and Rafael, have used their drawings to conceal information [8]. However, we still do not have any means to identify the real contents, or even intention, of these messages.

Sympathetic inks were a widespread technique. Who has not heard about lemon-based ink during childhood? With this type of ink, it is possible to write an innocent letter having a very different message written between its lines.

Science has developed new chemical substances that, combined with other substances, cause a reaction that makes the result visible. One of them is gallotanic acid, made from gall nuts, that becomes visible when coming in contact with copper sulfate [9].

With the continuous improvement of lenses, photo cameras, and films, people were able to reduce the size of a photo down to the size of a printed period $[7,8]$. One such example is micro-dot technology, developed by the Germans during the Second World War, referred to as the "enemy's masterpiece of espionage" by the FBI's director J. Edgar Hoover. Micro-dots are photographs the size of a printed period that have the clarity of standardsized typewritten pages. Generally, micro-dots were not hidden, nor encrypted messages. They were just so small as to not draw attention to themselves. The micro-dots allowed the transmission of large amounts of data (e.g., texts, drawings, and photographs) during the war.

There are also other forms of hidden communications, like null ciphers. Using such techniques, the real message is "camouflaged" in an innocuous message. The messages are very hard to construct and usually look like strange text. This strangeness factor can be reduced if the constructor has enough space and time. A famous case of a null cipher is the book Hypteronomachia Poliphili of 1499. A Catholic priest named Colona decided to declare his love to a young lady named Polya by putting the message "Father Colona Passionately loves Polia" in the first letter of each chapter of his book. 


\section{Social impacts}

Science and technology changed the way we lived in the $20^{\text {th }}$ century. However, this progress is not without risk. Evolution may have a high social impact, and digital Steganography is no different.

Over the past few years, Steganography has received a lot of attention. Since September $11^{\text {th }}, 2001$, some researchers have suggested that Osama Bin Laden and Al Qaeda used Steganography techniques to coordinate the World Trade Center attacks. Six years later, nothing was proved $[10,11,12,13]$. However, since then, Steganography has been a hype.

As a matter of fact, it is important to differentiate what is merely a suspicion from what is real - the hype or the hallelujah. There are many legal uses for Steganography and Steganalysis, as we show in Section 5. For instance, we can employ Steganography to create smart data structures and robust watermarking to track and authenticate documents, to communicate privately, to manage digital elections and electronic money, to produce advanced medical imagery, and to devise modern transit radar systems. Unfortunately, there are also illegal uses of these techniques. According to the High Technology Crimes Annual Report [14, 15], Steganography and Steganalysis can be used in conjunction with dozens of other cyber-crimes such as: fraud and theft, child pornography, terrorism, hacking, online defamation, intellectual property offenses, and online harassment. There are strong indications that Steganography has been used to spread child pornography pictures on the internet $[2,3]$.

In this work, we present some possible techniques and legal applications of Steganography and Steganalysis. Of course, the correct use of the information therein is all part of the reader's responsibility.

\section{Scientific and commercial applications}

In this section, we show that there are many applications for Information Hiding.

- Advanced data structures. We can devise data structures to conceal unplanned information without breaking compatibility with old software. For instance, if we need extra information about photos, we can put it in the photos themselves. The information will travel with the photos, but it will not disturb old software that does not know of its existence. Furthermore, we can devise advanced data structures that enable us to use small pieces of our hard disks to secretly conceal important information $[16,17]$.

- Medical imagery. Hospitals and clinical doctors can put together patient's exams, imagery, and their information. When a doctor analyzes a radiological exam, the patient's 
information is embedded in the image, reducing the possibility of wrong diagnosis and/or fraud. Medical-image steganography requires extreme care when embedding additional data within the medical images: the additional information must not affect the image quality $[18,19]$.

- Strong watermarks. Creators of digital content are always devising techniques to describe the restrictions they place on their content. These technique can be as simple as the message "Copyright 2007 by Someone" [20], as complex as the digital rights management system (DRM) devised by Apple Inc. in its iTunes store's contents [21], or the watermarks in the contents of the Vatican Library [22].

- Military agencies. Militaries' actions can be based on hidden and protected communications. Even with crypto-graphed content, the detection of a signal in a modern battlefield can lead to the rapid identification and attack of the involved parties in the communication. For this reason, military-grade equipment uses modulation and spread spectrum techniques in its communications [20].

- Intelligence agencies. Justice and Intelligence agencies are interested in studying these technologies, and identifying their weaknesses to be able to detect and track hidden messages $[23,2,3]$.

- Document tracking tools. We can use hidden information to identify the legitimate owner of a document. If the document is leaked, or distributed to unauthorized parties, we can track it back to the rightful owner and perhaps discover which party has broken the license distribution agreement [20].

- Document authentication. Hidden information bundled into a document can contain a digital signature that certifies its authenticity [20].

- General communication. People are interested in these techniques to provide more security in their daily communications [10,20]. Many governments continue to see the internet, corporations, and electronic conversations as an opportunity for surveillance [24].

- Digital elections and electronic money. Digital elections and electronic money are based on secret and anonymous communications techniques [5, 20].

- Radar systems. Modern transit radar systems can integrate information collected in a radar base station, avoiding the need to send separate text and pictures to the receiver's base stations.

- Remote sensing. Remote sensing can put together vector maps and digital imagery of a site, further improving the analysis of cultivated areas, including urban and natural sites, among others. 


\section{Steganography}

In this section, we present some of the most common techniques used to embed messages in digital images. We choose digital images as cover objects because they are more related to Computer Vision and Image Processing. However, these techniques can be extended to other types of digital media as cover objects, such as text, video, and audio files.

In general, steganographic algorithms rely on the replacement of some noise component of a digital object with a pseudo-random secret message [1]. In digital images, the most common noise component is the least significant bits (LSBs). To the human eye, changes in the value of the LSB are imperceptible, thus making it an ideal place for hiddhidinging information without any perceptual change in the cover object.

The original LSB information may have statistical properties, so changing some of them could result in the loss of those properties. Thus, we have to embed the message mimicking the characteristics of the cover bits' [9]. One possibility is to use a selection method in which we generate a large number of cover messages in the same way, and we choose the one having the secret embedded in it. However, this method is computationally expensive and only allows small embeddings. Another possibility is to use a constructive method. In this approach, we build a mimic function that also simulates characteristics of the cover bits noise.

Generally, both the sender and the receiver share a secret key and use it with a keystream generator. The key-stream is used for selecting the positions where the secret bits will be embedded [9].

Although LSB embedding methods hide data in such a way that humans do not perceive it, these embeddings often can be easily destroyed. As LSB embedding takes place on noise, it is likely to be modified, and destroyed, by further compression, filtering, or a less than perfect format or size conversion. Hence, it is often necessary to employ sophisticated techniques to improve embedding reliability as we describe in Section 6.3. Another possibility is to use techniques that take place on the most significant parts of the digital object used. These techniques must be very clever in order to not modify the cover object making the alterations imperceptible.

\subsection{LSB insertion/modification}

Among all message embedding techniques, LSB insertion/modification is a difficult one to detect [1, 20,13], and it is imperceptible to humans [20]. However, it is easy to destroy [25]. A typical color image has three channels: red, green and blue (R,G,B); each one offers one possible bit per pixel to the hiding process. 
In Figure 3, we show an example of how we can possibly hide information in the LSB fields. Suppose that we want to embed the bits $\mathbf{1 1 1 0}$ in the selected area. In this example, without loss of generality, we have chosen a gray-scale image, so we have one bit available in each image pixel for the hiding process. If we want to hide four bits, we need to select four pixels. To perform the embedding, we tweak the selected LSBs according to the bits we want to hide.

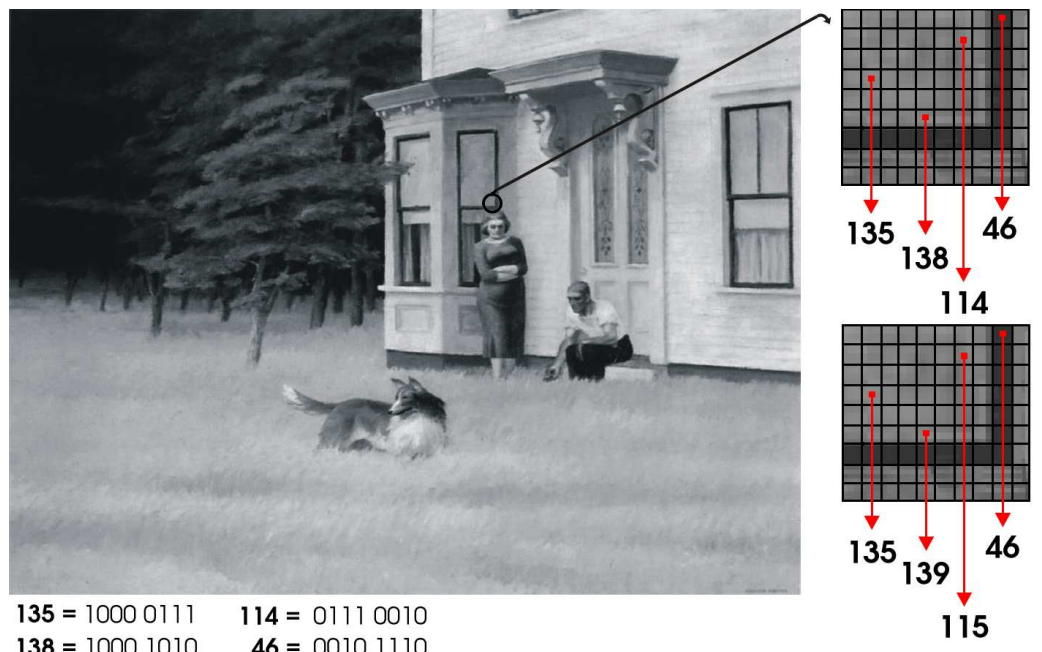

Figure 3. The LSB embedding process.

\subsection{FFTs and DCTs}

A very effective way of hiding data in digital images is to use a Direct Cosine Transform (DCT), or a Fast Fourier Transform (FFT), to hide the information in the frequency domain. The DCT algorithm is one of the main components of the JPEG compression technique [26]. In general, DCT and FFT work as follows:

1. Split the image into $8 \times 8$ blocks.

2. Transform each block via a DCT/FFT. This outputs a multi-dimensional array of 64 coefficients.

3. Use a quantizer to round each of these coefficients. This is essentially the compression stage and it is where data is lost. Small unimportant coefficients are rounded to 0 while 
larger ones lose some of their precision.

4. At this stage you should have an array of streamlined coefficients, which are further compressed via a Huffman encoding scheme or something similar.

5. To decompress, use the inverse DCT/FFT.

The hiding process using a DCT/FFT is useful because anyone that looks at pixel values of the image would be unaware that anything is different [20].

6.2.1 Least significant coefficients. It is possible to use LSB of the quantized DCT/FFT coefficients as redundant bits, and embed the hidden message there. The modification of a single DCT/FFT coefficient affects all 64 image pixels in the block [4]. Two of the simpler frequency-hiding algorithms are JSteg [27] and Outguess [28].

JSteg, Algorithm 1, sequentially replaces the least significant bit of DCT, or FFT, coefficients with the message's data. The algorithm does not use a shared key, hence, anyone who knows the algorithm can recover the message's hidden bits.

On the other hand, Outguess, Algorithm 2, is an improvement over JSteg, because it uses a pseudo-random number generator (PRNG) and a shared key as the PRNG's seed to choose the coefficients to be used.

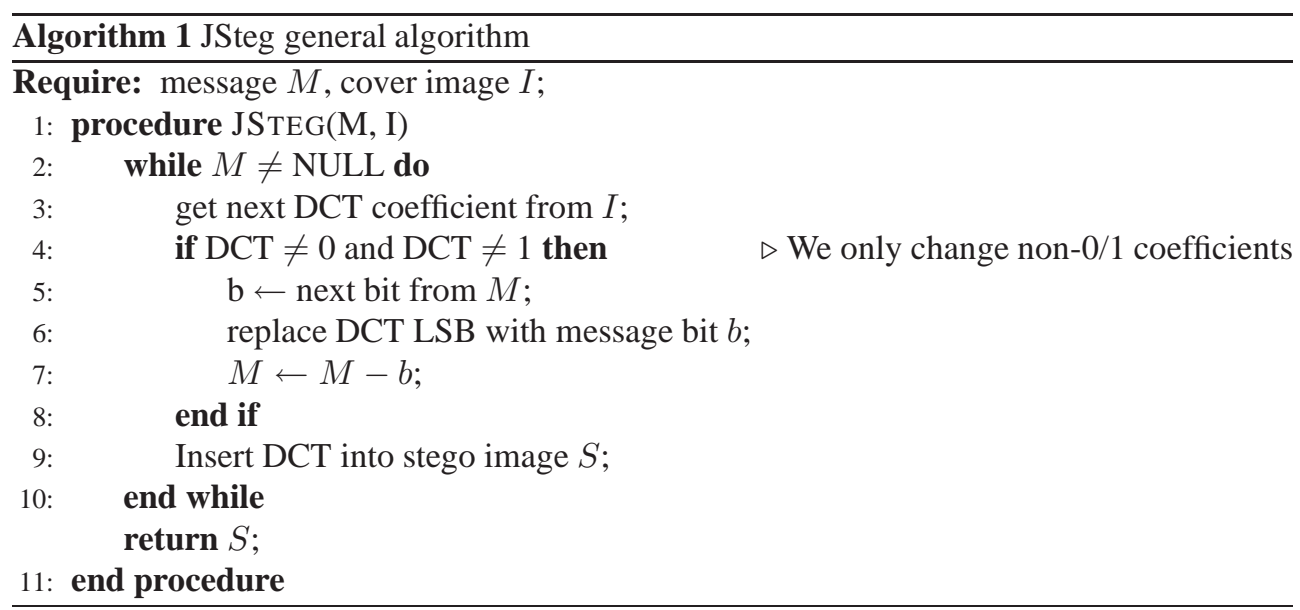

6.2.2 Block tweaking. It is possible to hide data during the quantization stage [20]. If we want to encode the bit value 0 in a specific $8 \times 8$ square of pixels, we can do this by making 


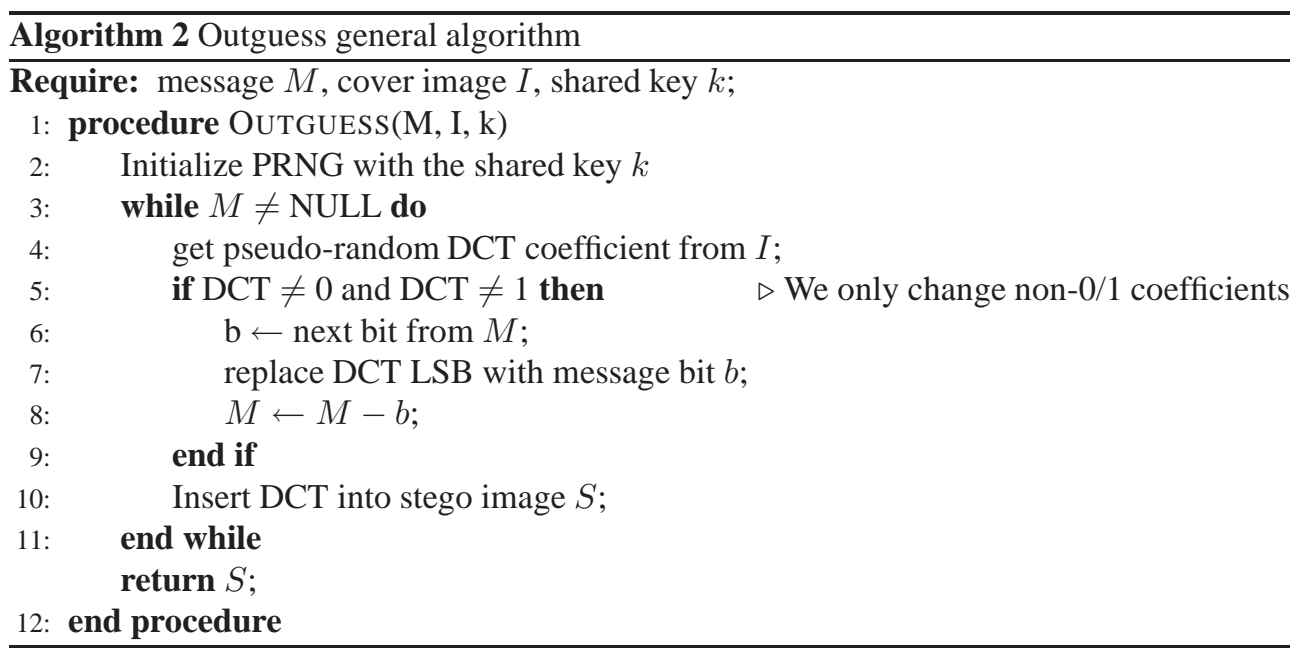

sure that all the coefficients are even in such a block, for example by tweaking them. In a similar approach, bit value 1 can be stored by tweaking the coefficients so that they are odd.

With the block tweaking technique, a large image can store some data that is quite difficult to destroy when compared to the LSB method. Although this is a very simple method and works well in keeping down distortions, it is vulnerable to noise $[20,1]$.

6.2.3 Coefficient selection. This technique consists of the selection of the $k$ largest DCT or FFT coefficients $\left\{\gamma_{1} \ldots \gamma_{k}\right\}$ and modify them according to a function $f$ that also takes into account a measure $\alpha$ of the required strength of the embedding process. Larger values of $\alpha$ are more resistant to error, but they also introduce more distortions.

The selection of the coefficients can be based on visual significance (e.g., given by zigzag ordering [20]). The factors $\alpha$ and $k$ are user-dependent. The function $f(\cdot)$ can be

$$
f\left(\gamma_{i}^{\prime}\right)=\gamma_{i}+\alpha b_{i},
$$

where $b_{i}$ is a bit we want to embed in the coefficient $\gamma_{i}$.

6.2.4 Wavelets. DCT/FFT transformations are not so effective at higher-compression levels. In such scenarios, we can use wavelet transformations instead of DCT/FFTs to improve robustness and reliability.

Wavelet-based techniques work by taking many wavelets to encode a whole image. They allow images to be compressed by storing the high and low frequency details separately 
in the image. We can use the low frequencies to compress the data, and use a quantization step to compress even more. Information hiding techniques using wavelets are similar to the ones with DCT/FFT [20].

\subsection{How to improve security}

Robust Steganography systems must observe the Kerckhoffs' Principle [29] in Cryptography, which holds that a cryptographic system's security should rely solely on the key material. Furthermore, to remain undetected, the unmodified cover medium used in the hiding process must be kept secret or destroyed. If it is exposed, a comparison between the cover and stego media immediately reveals the changes.

Further procedures to improve security in the hiding process are:

- Cryptography. Steganography supplements Cryptography, it does not replace it. If a hidden message is encrypted, it must also be decrypted if discovered, which provides another layer of protection [30].

- Statistical profiling. Data embedding alters statistical properties of the cover medium. To overcome such alterations, the embedding procedure can learn the statistics about the cover medium in order to minimize the amount of changes. For instance, for each bit changed to zero, the embedding procedure changes another bit to one.

- Structural profiling. Mimicking the statistics of a file is just the beginning. We can use the structure of the cover medium to better hide the information. For instance, if our cover medium is an image of a person, we can choose regions of this image that are rich in details such as the eyes, mouth and nose. These areas are more resilient to compression and conversion artifacts [26].

- Change of the order. Change the order in which the message is presented. The order itself can carry the message. For instance, if the message is a list of items, the order of the items can itself carry another message.

- Split the information. We can split the data into any number of packets and send them through different routes to their destination. We can apply sophisticated techniques in order to need only $k$ out of $n$ parts to reconstruct the whole message [20].

- Compaction. Less information to embed means fewer changes in the cover medium, lowering the probability of detection. We can use compaction to shrink the message and the amount of needed alterations in the cover medium. 


\section{Steganalysis}

With the indications that steganography techniques have been used to spread child pornography pictures on the internet $[2,3]$, there is a need to design and evaluate powerful detection techniques able to avoid or minimize such actions. In this section, we present an overview of current approaches, attacks, and statistical techniques available in Steganalysis.

Steganalysis refers to the body of techniques devised to detect hidden contents in digital media. It is an allusion to Cryptanalysis which refers to the body of techniques devised to break codes and cyphers [29].

In general, it is enough to detect whether a message is hidden in a digital content. For instance, law enforcement agencies can track access logs of hidden contents to create a network graph of suspects. Later, using other techniques, such as physical inspection of apprehended material, they can uncover the actual contents and apprehend the guilty parties [13,30]. There are three types of Steganalysis attacks: (1) aural; (2) structural; and (3) statistical.

1. Aural attacks. They consist of striping away the significant parts of a digital content in order to facilitate a human's visual inspection for anomalies [20]. A common test is to show the LSBs of an image.

2. Structural attacks. Sometimes, the format of the digital file changes as hidden information is embedded. Often, these changes lead to an easily detectable pattern in the structure of the file format. For instance, it is not advisable to hide messages in images stored in GIF format. In such a format an image's visual structure exists to some degree in all of an image's bit layers due to the color indexing that represents $2^{24}$ colors using only 256 values [31].

3. Statistical attacks. Digital pictures of natural scenes have distinct statistical behavior. With proper statistical analysis, we can determine whether or not an image has been altered, making forgeries mathematically detectable [23]. In this case, the general purpose of Steganalysis is to collect sufficient statistical evidence about the presence of hidden messages in images, and use them to classify [32] whether or not a given image contains a hidden content. In the following section, we present some available statistical-based techniques for hidden message detection.

\section{1 $\chi^{2}$ analysis}

Westfeld and Pfitzmann [31] have present $\chi^{2}$ analysis to detect hidden messages. They showed that an $L$-bit color channel can represent $2^{L}$ possible values. If we split these values into $2^{L-1}$ pairs which only differ in the LSBs, we are considering all possible patterns of 
neighboring bits for the LSBs. Each of these pairs is called a pair of value (PoV) in the sequence [31].

When we use all the available LSB fields to hide a message in an image, the distribution of odd and even values of a PoV will be the same as the 0/1 distribution of the message bits. The idea of the $\chi^{2}$ analysis is to compare the theoretically expected frequency distribution of the PoVs with the real observed ones [31]. However, we do not have the original image and thus the expected frequency. In the original image, the theoretically expected frequency is the arithmetical mean of the two frequencies in a PoV. As we know, the embedding function only affects the LSBs, so it does not affect the PoV's distribution after an embedding. Given that, the arithmetical mean remains the same in each PoV, and we can derive the expected frequency through the arithmetic mean between the two frequencies in each PoV.

Westfeld and Pfitzmann [31] have showed that we can apply the $\chi^{2}$ (chi squared-test) over these PoVs to detect hidden messages. The $\chi^{2}$ test general formula is

$$
\chi^{2}=\sum_{i=1}^{\nu+1} \frac{\left(f_{i}^{o b s}-f_{i}^{e x p}\right)^{2}}{f_{i}^{e x p}},
$$

where $\nu$ is the number of analyzed PoVs, $f_{i}^{o b s}$ and $f_{i}^{e x p}$ are the observed frequencies and the expected frequencies respectively.

The probability of hiding, $p h$, in a region is given by the compliment of the cumulative distribution

$$
p h=1-\int_{0}^{\chi^{2}} \frac{t^{(\nu-2) / 2} e^{-t / 2}}{2^{\nu / 2} \Gamma(\nu / 2)} d t,
$$

where $\Gamma(\cdot)$ is the Euler-Gamma function. We can calculate this probability in different regions of the image.

This approach can only detect sequential messages hidden in the first available pixels' LSBs, as it only considers the descriptors' value. It does not take into account that, for different images, the threshold value for detection may be quite distinct [13].

Simply measuring the descriptors constitutes a low-order statistic measurement. This approach can be defeated by techniques that maintain basic statistical profiles in the hiding process $[13,33]$.

Improved techniques such as Progressive Randomization (PR) [13] addresses the loworder statistics problem by looking at the descriptors' behavior along selected regions (feature regions). 


\subsection{RS analysis}

Fridrich et al. have presented RS analysis [34]. It consists of the analysis of the LSB loss-less embedding capacity in color and gray-scale images. The loss-less capacity reflects the fact that the LSB plane - even though it looks random - is related to the other bit planes [34]. Modifications in the LSB plane can lead to statistically detectable artifacts in the other bit planes of the image.

To measure this behavior, Fridrich and colleagues have proposed simulation of artificial new embeddings in the analyzed images using some defined functions.

Let $I$ be the image to be analyzed with width $W$ and height $H$ pixels. Each pixel has values in $P$. For an 8 bits per pixel image, we have $P=\{0 \ldots 255\}$. We divide $I$ into $G$ disjoint groups of $n$ adjacent pixels. For instance, we can choose $n=4$ adjacent pixels. We define a discriminant function $f$ responsible to give a real number $f\left(x_{1}, \ldots, x_{n}\right) \in \Re$ for each group of pixels $G=\left(x_{1}, \ldots, x_{n}\right)$. Our objective using $f$ is to capture the smoothness of $G$. Let the discrimination function be

$$
f\left(x_{1}, \ldots, x_{n}\right)=\sum_{i=1}^{n-1}\left|x_{i+1}-x_{i}\right| .
$$

Furthermore, let $F_{1}$ be a flipping invertible function $F_{1}: 0 \leftrightarrow 1,2 \leftrightarrow 3, \ldots, 254 \leftrightarrow 255$, and $F_{-1}$ be a shifting function $F_{-1}:-1 \leftrightarrow 0,1 \leftrightarrow 2, \ldots, 255 \leftrightarrow 256$ over $P$. For completeness, let $F_{0}$ be the identity function such as $F_{0}(x)=x \forall x \in P$.

Define a mask $\mathcal{M}$ that represents which function to apply to each element of a group $G$. The mask $\mathcal{M}$ is an $n$-tuple with values in $\{-1,0,1\}$. The value -1 stands for the application of the function $F_{-1} ; 1$ stands for the function $F_{1}$; and 0 stands for the identity function $F_{0}$. Similarly, we define $-\mathcal{M}$ as $\mathcal{M}$ 's compliment.

We apply the discriminant function $f$ with the functions $F_{\{-1,0,1\}}$ defined through a mask $\mathcal{M}$ over all $G$ groups to classify them into three categories:

- Regular. $G \in R_{\mathcal{M}} \Leftrightarrow f\left(F_{\mathcal{M}}(G)\right)>f(G)$

- Singular. $G \in S_{\mathcal{M}} \Leftrightarrow f\left(F_{\mathcal{M}}(G)\right)<f(G)$

- Unusable. $G \in U_{\mathcal{M}} \Leftrightarrow f\left(F_{\mathcal{M}}(G)\right)=f(G)$

Similarly, we classify the groups $R_{-\mathcal{M}}, S_{-\mathcal{M}}$, and $U_{-\mathcal{M}}$ for the mask $-\mathcal{M}$. As a matter of fact, it holds that

$$
\frac{R_{\mathcal{M}}+S_{\mathcal{M}}}{T} \leq 1 \quad \text { and } \quad \frac{R_{-\mathcal{M}}+S_{-\mathcal{M}}}{T} \leq 1,
$$


where $T$ is the total number of $G$ groups.

The method's statistical hypothesis is that, for typical images

$$
R_{\mathcal{M}} \approx R_{-\mathcal{M}} \quad \text { and } \quad S_{\mathcal{M}} \approx S_{-\mathcal{M}} .
$$

What is interesting is that, in an image with a hidden content, the greater the message size, the greater the $R_{-\mathcal{M}}$ and $S_{-\mathcal{M}}$ difference, and the lower the difference between $R_{\mathcal{M}}$ and $S_{\mathcal{M}}$. This behavior points out to high-probability chance of embedding in the analyzed image [34].

\subsection{Gradient-energy flipping rate}

Li Shi et al. have presented the Gradient-Energy Flipping Rate (GEFR) technique for Steganalysis. It consists in the analysis of the gradient-energy variation due to the hiding process [35].

Let $I(n)$ be an unidimensional signal. The gradient $r(n)$, before the hiding is

$$
r(n)=I(n)-I(n-1),
$$

and the $I(n)$ 's gradient energy (GE), is

$$
G E=\sum|I(n)-I(n-1)|^{2}=\sum r(n)^{2} .
$$

After the hiding of a signal $S(n)$ in the original signal, $I(n)$ becomes $I^{\prime}(n)$ and the gradient becomes

$$
\begin{aligned}
r(n) & =I(n)-I(n-1) \\
& =(I(n)+S(n))-(I(n-1)+S(n-1)) \\
& =r(n)+S(n)-S(n-1) .
\end{aligned}
$$

The probability distribution function of $S(n)$ is

$$
\left\{\begin{array}{l}
\rho(S(n)) \approx 0=\frac{1}{2} \\
\rho(S(n)) \approx \pm 1=\frac{1}{4}
\end{array}\right.
$$

After any kind of embedding, the new gradient energy $G E^{\prime}$ is

$$
\begin{aligned}
G E^{\prime} & =\sum|r(n)|^{2}=\sum|r(n)+S(n)-S(n-1)|^{2} \\
& =\sum|r(n)+\Delta(n)|^{2}, \text { where } \Delta(n)=S(n)-S(n-1) .
\end{aligned}
$$

To perform the detection, it is necessary to define a process of inverting the bits of an image's LSB plane. For that, we can use a function $F$ which is similar to the one we described in Section 7.2. 
Let $I$ be the cover image with $W \times H$ pixels and $p \leq W \times H$ be the size of the hidden message. The application of the function $F$ results in the properties:

- For $p=W \times H$, there is $\frac{W \times H}{2}$ pixels with inverted LSB. That means that the embedding rate is $50 \%$ and the gradient energy is given by $G E=\left(\frac{W \times H}{2}\right)$.

- The original image's gradient energy is given by $E G(0)$. After inverting all available LSBs using $F$, the gradient energy becomes $G E^{\prime}=W \times H$.

- For $p<W \times H$, there is $\frac{p}{2}$ pixels with inverted LSB. Let $I\left(\frac{p}{2}\right)$ be the modified image. The resulting gradient energy is $G E=\frac{p / 2}{W \times H}=E G(0)+p$. If $F$ is applied over $I\left(\frac{p}{2}\right)$, the resulting gradient energy is $E G=\frac{W \times H-p / 2}{W \times H}$.

With these properties, Li Shi et al. have proposed the following detection procedure:

1. Find the test image's gradient energy $G E\left(\frac{p / 2}{W \times H}\right)$;

2. Apply $F$ over the test image and calculate $G E\left(\frac{W \times H-p / 2}{W \times H}\right)$;

3. Find $G E\left(\frac{W \times H}{2}\right)=\left[E G\left(\frac{p / 2}{W \times H}\right)+G E\left(\frac{W \times H-p / 2}{W \times H}\right)\right] / 2$;

4. $G E(0)$ is based on $G E\left(\frac{W \times H}{2}\right)=G E(0)+W \times H$;

5. Finally, the estimated size of the hidden message is given by

$$
p^{\prime}=G E\left(\frac{p / 2}{W \times H}\right)-G E(0) .
$$

\subsection{High-order statistical analysis}

Lyu and Farid [36, 37, 38, 39] have introduced a detection approach based on highorder statistical descriptors. Natural images have regularities that can be detected by highorder statistics through wavelet decompositions [38]. To decompose the images, Lyu and 
colleagues have used quadrature mirror filters (QMFs) [40]. This decomposition divides the image into multiple scales and orientations resulting in four subbands: vertical, horizontal, diagonal, and low-pass which can be recursively used to produce subsequent scales.

Let $V_{i}(x, y), H_{i}(x, y)$, and $D_{i}(x, y)$ be the vertical, horizontal, and diagonal subbands for a given scale $i \in\{1 \ldots n\}$. Figure 4 depicts this process.

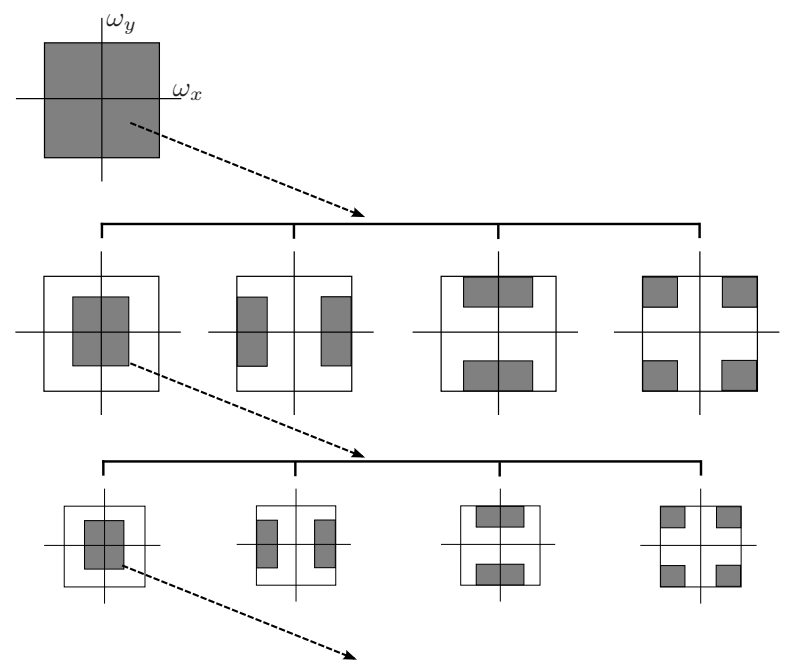

Figure 4. QMF decomposition scheme.

From the QMF decomposition, the authors create a statistical model composed of mean, variance, skewness, and kurtosis for all subbands and scales. These statistics characterize the basic coefficients' distribution. The second set of statistics is based on the errors in an optimal linear predictor of coefficient magnitude. The subband coefficients are correlated to their spatial, orientation, and scale neighbors [41]. For illustration purposes, consider first a vertical band, $V_{i}(x, y)$, at scale $i$. A linear predictor for the magnitude of these coefficients in a subset of all possible neighbors is given by

$$
\begin{aligned}
V_{i}(x, y)= & w_{1} V_{i}(x-1, y)+w_{2} V_{i}(x+1, y)+w_{3} V_{i}(x, y-1)+w_{4} V_{i}(x, y+1)+ \\
& +w_{5} V_{i+1}\left(\frac{x}{2}, \frac{y}{2}\right)+w_{6} D_{i}(x, y)+w_{7} D_{i+1}\left(\frac{x}{2}, \frac{y}{2}\right),
\end{aligned}
$$

where $w_{k}$ denotes the scalar weighting values. The error coefficients are calculated using quadratic minimization of the error function

$$
E(w)=[V-Q w]^{2},
$$


where $w=\left(w_{1}, \ldots, w_{7}\right)^{T}, V$ is a column vector of magnitude coefficients, and $Q$ is the magnitude neighbors' coefficients as proposed in Equation 10. The error function is minimized through differentiation with respect to $w$

$$
\frac{d E(w)}{d w}=2 Q^{T}[V-Q w] .
$$

After simplifications, we calculate $w_{k}$ directly with the linear predictor log error

$$
E=\log _{2}(V)-\log _{2}(|Q w|) .
$$

With a recursive application of this process to all subbands, scales, and orientation, we have a total of $12(n-1)$ error statistics plus $12(n-1)$ basic ones. This amounts to a $24(n-1)$ sized feature vector. This feature vector feeds a classifier, which is able to output whether or not an unknown image contains a hidden message. Lyu and colleagues have used Linear Discriminant Analysis and Support Vector Machines to perform the classification stage [32].

\subsection{Image quality metrics}

Avcibas et al. have presented a detection scheme based on image quality metrics (IQMs) [42, 43, 44, 45]. Image quality metrics are often used for coding artifact evaluation, performance prediction of vision algorithms, quality loss due to sensor inadequacy, etc.

Steganographic schemes, whether by spread-spectrum, quantization modulation, or LSB insertion/modification, can be represented as a signal addition to the cover image. In this context, Avcibas and colleagues' hypothesis is that steganographic schemes leave statistical evidences that can be exploited for detection with the aid of IQMs and multivariate regression analysis (ANOVA).

Using ANOVA, the authors have pointed out that the following IQMs are the best feature generators: mean absolute error, mean square error, Czekznowski correlation, image fidelity, cross correlation, spectral magnitude distance, normalized mean square, HVS error, angle mean, median block spectral phase distance, and median block weighted spectral distance.

After measuring the IQMs in a training set of images with and without hidden messages, the authors propose a multivariate normalized regression to values -1 and 1 . In the regression model, each decision is expressed by $y_{i}$ in a set of $n$ observation images and $q$ 
available IQMs. A linear function of the IQMs is given by

$$
\left\{\begin{aligned}
y_{1} & =\beta_{1} x_{11}+\beta_{2} x_{12}+\ldots+\beta_{q} x_{1 q}+\epsilon_{1} \\
y_{2} & =\beta_{2} x_{21}+\beta_{2} x_{22}+\ldots+\beta_{q} x_{2 q}+\epsilon_{2} \\
& \vdots \\
y_{N} & =\beta_{n} x_{n 1}+\beta_{2} x_{12}+\ldots+\beta_{q} x_{n q}+\epsilon_{n},
\end{aligned}\right.
$$

where $x_{i j}$ is the quality coefficient for the image $i \in\{1 \ldots n\}$ and IQM $j \in\{1 \ldots q\}$. Finally, $\beta_{k}$ is the regression coefficient, and $\epsilon$ is random error.

Once we calculate these coefficients, we can use the resulting coefficient vector to any new image in order to classify it as stego or non-stego image.

\subsection{Progressive Randomization (PR)}

Rocha and Goldenstein $[13,25]$ have presented the Progressive Randomization descriptor for Steganalysis. It is a new image descriptor that captures the difference between image classes (with and without hidden messages) using the statistical artifacts inserted during a perturbation process that increases randomness with each step.

Algorithm 3 summarizes the four stages of PR applied to Steganalysis: the randomization process (Section 7.6.2); the selection of feature regions (Section 7.6.3); the statistical descriptors analysis (Section 7.6.4), and invariance (Section 7.6.5).

7.6.1 Pixel perturbation. Let $\mathbf{x}$ be a Bernoulli distributed random variable with $\operatorname{Prob}\{\mathbf{x}=$ $0\})=\operatorname{Prob}(\{\mathbf{x}=1\})=1 / 2, B$ be a sequence of bits composed by independent trials of $\mathbf{x}$, $p$ be a percentage, and $S$ be a random set of pixels of an input image.

Given an input image $I$ of $|I|$ pixels, we define the LSB pixel perturbation $T(I, p)$ the process of substitution of the LSBs of $S$ of size $p \times|I|$ according to the bit sequence $B$. Consider a pixel $p x_{i} \in S$ and an associated bit $b_{i} \in B$

$$
\mathcal{L}\left(p x_{i}\right) \leftarrow b_{i} \text { for all } p x_{i} \in S .
$$

where $\mathcal{L}\left(p x_{i}\right)$ is the LSB of the pixel $p x_{i}$.

7.6.2 The randomization process. Given an original image $I$ as input, the randomization process consists of the progressive application $I, T\left(I, P_{1}\right), \ldots, T\left(I, P_{n}\right)$ of LSB pixel disturbances. The process returns $n$ images that only differ in the LSB from the original image and are identical to the naked eye.

The $T\left(I, P_{i}\right)$ transformations are perturbations of different percentages of the available LSBs. Here, we use $n=6$ where $P=\{1 \%, 5 \%, 10 \%, 25 \%, 50 \%, 75 \%\}, P_{i} \in P$ 
Algorithm 3 The PR descriptor

Require: Input image $I$; Percentages $P=\left\{P_{1}, \ldots P_{n}\right\}$;

1: Randomization: perform $n$ LSB pixel disturbances of the original image $\triangleright$ Sec. 7.6.2

$$
\left\{O_{i}\right\}_{i=0 \ldots n .}=\left\{I, T\left(I, P_{1}\right), \ldots, T\left(I, P_{n}\right)\right\} .
$$

2: Region selection: select $r$ feature regions of each image $i \in\left\{O_{i}\right\}_{i=0 \ldots n} \quad \triangleright$ Sec. 7.6.3

$$
\left\{O_{i j}\right\}_{\substack{i=0 \ldots n, j=1 \ldots r .}}^{\substack{i=n \\ j}}=\left\{O_{01}, \ldots, O_{n r}\right\} .
$$

3: Statistical descriptors: calculate $m$ descriptors for each region

$$
\left\{d_{i j k}\right\}=\left\{d_{k}\left(O_{i j}\right)\right\} \begin{array}{r}
i=0 \ldots n, \\
j=1 \ldots r \\
k=1 \ldots m .
\end{array}
$$

4: Invariance: normalize the descriptors based on $I$

$$
F=\left\{f_{e}\right\}_{e=1 \ldots n \times r \times m}=\left\{\frac{d_{i j k}}{d_{0 j k}}\right\}_{\begin{array}{l}
i=0 \ldots n \\
j=1 \ldots r \\
k=1 \ldots m
\end{array}}
$$

5: Classification. Use $F \in \Re^{n \times r \times m}$ in your favorite machine learning black box.

denotes the relative sizes of the set of selected pixels $S$. The greater the LSB pixel disturbance, the greater the resulting LSB entropy of the transformation.

7.6.3 Feature region selection. Local image properties do not show up under a global analysis [20]. The authors use statistical descriptors of local regions to capture the changing dynamics of the statistical artifacts inserted during the randomization process (Section 7.6.2).

Given an image $I$, they use $r$ regions with size $l \times l$ pixels to produce localized statistical descriptors (Figure 5).

7.6.4 Statistical descriptors. When we disturb all the available LSBs in $S$ with a sequence $B$, the distribution of 0/1 values of a PoV (see Section 7.1) will be the same as in $B$. The authors apply the $\chi^{2}$ (chi-squared test) [31] and $U_{T}$ (Ueli Maurer Universal Test) [46] to analyze the images.

- $\chi^{2}$ test. The $\chi^{2}$ test [47] compares two histograms $f^{\text {obs }}$ and $f^{e x p}$. Histogram $f^{o b s}$ represents the observations and $f^{\exp }$ represents the expected histogram. The procedure 

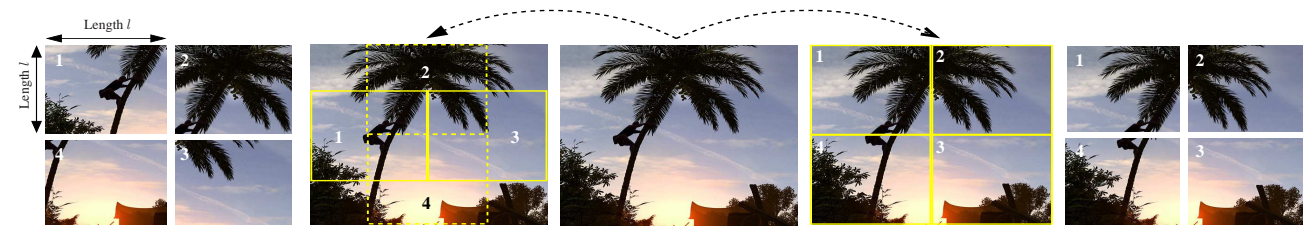

Figure 5. The PR eight overlapping regions.

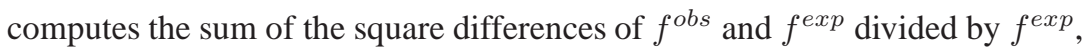

$$
\chi^{2}=\sum_{i} \frac{\left(f_{i}^{o b s}-f_{i}^{e x p}\right)^{2}}{f_{i}^{e x p}} .
$$

- Ueli test. The Ueli test $\left(U_{T}\right)$ [46] is an effective way to evaluate the randomness of a given sequence of numbers. $U_{T}$ splits an input data $S$ into $n$ blocks. For each block $b_{i}$, it analyzes each of the $n-1$ remaining blocks, looks for the most recent occurrence of $b_{i}$, and takes the log of the summed temporal occurrences. Let $B(S)=$ $\left(b_{1}, b_{2}, \ldots, b_{N}\right)$ be a set of $n$ blocks such that $\cup_{\forall b_{i}}=S$. Let $\left|b_{i}\right|=L$ be the block size for each $i$ and $|B(S)|=N$ be the number of blocks. We define $U_{T}: B(S) \rightarrow \Re^{+}$as

$$
U_{T}(B(S))=\frac{1}{K} \sum_{i=Q}^{Q+K} \ln A\left(b_{i}\right)
$$

where $K$ is the number of analyzed bits (e.g., $K=N$ ), $Q$ is a shift in $B(S)$ (e.g., $\left.Q=\frac{K}{10}[46]\right)$, and

$$
A\left(b_{i}\right)= \begin{cases}i & \nexists i^{\prime} \in \mathbb{N}, i^{\prime}<i \mid b_{i^{\prime}}=b_{i} \\ \min \left\{i^{\prime}: b_{i^{\prime}}=b_{i}\right\} & \text { otherwise. }\end{cases}
$$

7.6.5 Invariance transformation. The variation rate of the statistical descriptors is more interesting than their values. The authors propose the normalization of all descriptors from the transformations with regard to their values in the original image $I$

$$
F=\left\{f_{e}\right\}_{e=1 \ldots n \times r \times m}=\left\{\frac{d_{i j k}}{d_{0 j k}}\right\}_{\begin{array}{l}
i=0 \ldots n \\
j=1 \ldots r \\
k=1 \ldots m
\end{array}}
$$

where $d$ denotes a descriptor $1 \leq k \leq m$ of a region $1 \leq j \leq r$ of an image $0 \leq i \leq n$, and $F$ is the final generated descriptor vector of the image $I$. 
7.6.6 Classification. The authors use a labeled set of images to learn the behavior of the selected statistical descriptors and train different classifiers (supervised learning). The goal is to determine whether a new incoming image contains a hidden message. They have trained and validated the technique using a series of classifiers such as CTREES, SVMS, LDA and Bagging ensembles [13, 25].

The statistical hypothesis is that the greater the embedded message, the lower the ratio between subsequent iterations of the progressive randomization operation. Images with no hidden content have different behavior under PR than images that have suffered some process of message embedding [13, 25].

\section{Freely available tools and software}

Many Steganography and Steganalysis applications are freely available on the internet for a great variety of platforms which includes DOS, Windows, Mac OS, Unix, and Linux.

Romana Machado has introduced Ezstego and Stego Online ${ }^{5}$, two tools designed in Java language suitable to Steganography in 8-bits indexed images stored in the GIF format [48].

Henry Hastur has presented two other tools: Mandelsteg e Stealth ${ }^{6}$. Mandelsteg generates fractal images to hide the messages. Stealth is a software that uses PGP Cryptography [49] in the embedding process. Two other software tools that incorporate Cryptography in the hiding process are White Noise Storm ${ }^{7}$ by Ray Arachelian and S-Tools ${ }^{8}$.

Colin Maroney has devised Hide and Seek ${ }^{9}$. This tool is able to hide a list of files in one image. However, it does not use Cryptography. Derek Upham has presented Jsteg ${ }^{10}$, which is able to hide messages using the DCT/FFT transformed space. Niels Provos has introduced Outguess ${ }^{11}$ which is an improvement over JSteg-based techniques.

Finally, Anderson Rocha and colleagues have introduced Camaleão ${ }^{12}$ [50, 51, 52], which uses cyclic permutations and block cyphering to hide messages in the least significant bits of loss-less compression images.

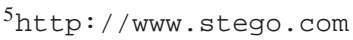

${ }^{6}$ ftp://idea.sec.dsi.unimi.it/pub/security/crypt/code/

${ }^{7}$ ftp. csua.berkeley.edu/pub/cypherpunks/steganography/wns210.zip

${ }^{8}$ ftp://idea.sec.dsi.unimi.it/pub/security/crypt/code/s-tools4.zip

${ }^{9}$ ftp: / / csua.berkeley.edu/pub/cypherpunks/steganography/hdsk41b.zip

${ }^{10}$ ftp. funet.fi/pub/crypt/steganography

${ }^{11}$ http: //www. outguess.org/

12 http: //andersonrocha.cjb. net
} 


\section{Open research topics}

When performing data-hiding in digital images, we have an additional problem: images are expected to be subjected to many operations, ranging from simple transformations, such as translations, to nonlinear transformations, such as blurring, filtering, lossy compression, printing, and rescanning. The hidden messages should survive all attacks that do not degrade the image's perceived quality [1].

Steganography's main problem involves designing robust information-hiding techniques. It is crucial to derive approaches that are robust to geometrical attacks as well as nonlinear transformations, and to find detail-rich regions in the image that do not lead to artifacts in the hiding process. The hidden messages should not degrade the perceived quality of the work, implying the need for good image-quality metrics.

Hiding techniques often rely on private key sharing, which involves previous communication. It is important to work on algorithms that use asymmetric key schemes.

If multiple messages are inserted in a single object, they should not interfere with each other [1].

We need new powerful Steganalysis techniques that can detect messages without prior knowledge of the hiding algorithm (blind detection). The detection of very small messages is also a significant problem. Finally, we need adaptive techniques that do not involve complex training stages.

\section{Conclusions}

In this tutorial, we have presented an overview of the past few years of Steganography and Steganalysis, we have showed some of the most interesting hiding and detection techniques, and we have discussed a series of applications on both topics.

Terrorism has infiltrated the public's perception of this technology for a long period. Public fear created by mainstream press reports, which often featured US intelligence agents claiming that terrorists were using Steganography, created a mystique around data hiding techniques. Legislators in several US states have either considered or passed laws prohibiting the use and dissemination of technology to conceal data [53].

Six years after September $11^{\text {th }}, 2001$ 's tragic incidents, Steganography and Steganalysis have become mature disciplines, and data hiding approaches have outlived their period of hype. Public perception should now move beyond the initial notion that these techniques are suitable only for terrorist-cells' communications. Steganography and Steganalysis have many legitimate applications, and represent great research opportunities waiting to be addressed. 


\section{Acknowledgments}

We thank the support of FAPESP (05/58103-3 and 07/52015-0) and CNPq (301278/2004 and 551007/2007-9). We also thank Dr. Valerie Miller for proof reading this article.

\section{References}

[1] R. Anderson and F. Petitcolas. On the limits of steganography. IEEE Journal of Selected Areas in Communications, 16:474-481, may 1998.

[2] Sara V. Hart, John Ashcroft, and Deborah J. Daniels. Forensic examination of digital evidence: a guide for law enforcement. Technical Report NCJ 199408, U.S. Department of Justice - Office of Justice Programs, Apr 2004.

[3] Sheridan Morris. The future of netcrime now (1) - threats and challenges. Technical Report 62/04, Home Office Crime and Policing Group, 2004.

[4] Niels Provos and Peter Honeyman. Hide and seek: an introduction to steganography. IEEE Security \& Privacy Magazine, 1:32-44, May 2003.

[5] Andreas Pfitzmann. Information hiding terminology. In Proceedings of the First Intl. Workshop on Information Hiding, Cambridge, UK, May 1996. Springer-Verlag.

[6] Fabien A. P. Petitcolas, Ross J. Anderson, and Markus G. Kuhn. Information hiding A survey. Proceedings of the IEEE, 87:1062-1078, Jul 1999.

[7] Bruce Norman. Secret warfare, the battle of Codes and Ciphers. Acropolis Books Inc., first edition, 1980. ISBN 0-87491-600-3.

[8] Marcus G. Kuhn. The history of steganography. In Proceedings of the First Intl. Workshop on Information Hiding, Cambridge, UK, May 1996. Springer-Verlag.

[9] Richard Popa. An analysis of steganography techniques. Master's thesis, The "Polytechnic" University of Timisoara, Timisoara, Romênia, 1998.

[10] Paul Wallich. Getting the message. In IEEE Spectrum, volume 40, pages 38-43, April 2003.

[11] Stephen Cass. Listening in. In IEEE Spectrum, volume 40, pages 32-37, April 2003.

[12] Jean Kumagai. Mission impossible? In IEEE Spectrum, volume 40, pages 26-31, April 2003. 
[13] Anderson Rocha and Siome Goldenstein. Progressive Randomization for Steganalysis. In $8^{\text {th }}$ IEEE Intl. Conf. on Multimedia and Signal Processing, 2006.

[14] USPS. USPS - US Postal Inspection Service. At www . usps . com/ postalinspectors/ar01intr.pdf, 2003.

[15] NHTCU. NHTCU - National High Tech Crime Unit. At www . nhtcu . org, 2003.

[16] H. Pang, K. L. Tan, and X. Zhou. StegFS: a steganographic file system. In $19^{\text {th }}$ Intl. Conference on Data Engineering, pages 657-667, March 2003.

[17] Steven Hand and Timothy Roscoe. Mnemosyne: Peer-to-peer steganographic storage. In $1^{\text {st }}$ Intl. Workshop on Peer-to-Peer Systems, volume 2429, pages 130-140, March 2002 .

[18] Raúl Rodríguez-Colín, Feregrino-Uribe Claudia, and Gershom de J. Trinidad-Blas. Data hiding scheme for medical images. In $17^{\text {th }}$ IEEE Intl. Conference on Electronics, Communications and Computers, pages 33-38, February 2007.

[19] Y. Li, C. T. Li, and C. H. Wei. Protection of mammograms using blind staganography and watermarking. In $3^{\text {rd }}$ Intl. Symposium on Information Assurance and Security, August 2007.

[20] Peter Wayner. Disappearing cryptography. Morgan Kaufmann Publishers, San Francisco, CA, USA, second edition, 2002. ISBN 1-55860-769-2.

[21] The Electronic Frontier Foundation (EFF). The customer is always wrong: A user's guide to DRM in online music. At http://www.eff.org/IP/DRM/guide/, 2007.

[22] F. C. Mintzer, L. E. Boyle, and A. N. Cases. Toward on-line, worldwide access to vatican library materials. IBM Journal of Research and Development, 40:139-162, Mar 1996.

[23] Rebecca T. Mercuri. The many colors of multimedia security. Communications of the ACM, 47:25-29, 2004.

[24] Toby Sharp. An implementation of key-based digital signal steganography. In $4^{\text {th }}$ Intl. Information Hiding Workshop, 2001.

[25] Anderson Rocha. Randomização Progressiva Para Esteganálise. Master's thesis, Instituto de Computação - Unicamp, Campinas, SP, Brasil, 2006.

[26] Rafael C. Gonzalez and Richard E. Woods. Digital Image Processing. Prentice-Hall, Boston, MA, USA, second edition, 2002. ISBN 0-20118-075-8. 
[27] Derek Upham. Jsteg shell. At http://www.tiac.net/users/korejwa/ jstegshella.zip, 1999.

[28] Niels Provos. Defending against statistical steganalysis. In Proceedings of the $10^{\text {th }}$ USENIX Security Symposium, pages 323-336, Washington, DC, USA, Aug 2001. The USENIX Association.

[29] Bruce Schneier. Applied Cryptography. John Wiley \& Sons, New York, 1995. ISBN 047111-709-9.

[30] Neil F. Johnson and Sushil Jajodia. Exploring steganography: Seeing the unseen. IEEE Computer, 31:26-34, Feb 1998.

[31] Andreas Westfeld and Andreas Pfitzmann. Attacks on steganographic systems. In Proceedings of the Third Intl. Workshop on Information Hiding, pages 61-76, London, UK, 1999. Springer Verlag.

[32] Christopher M. Bishop. Pattern Recognition and Machine Learning. Springer Verlab, 2006. ISBN 0-38731-073-8.

[33] Niels Provos and Peter Honeyman. Detecting steganographic content on the internet. Technical Report CITI 01-11, University of Michigan, Ann Arbor, MI, USA, Nov 2001.

[34] Jessica Fridrich, Miroslav Goljan, and Rui Du. Detecting LSB steganography in color and grayscale images. IEEE Multimedia, 8:22-28, Jan 2001.

[35] Li Shi, Sui Ai Fen, and Yang Yi Xian. A LSB steganography detection algorithm. In Proceedings of the $14^{\text {th }}$ Personal, Indoor and Mobile Radio Communications, volume 3, pages 2780-2783. IEEE, Sep 2003.

[36] Siwei Lyu and Hany Farid. Detecting hidden messages using higher-order statistics and support vector machines. In Proceedings of the Fifth Intl. Workshop on Information Hiding, pages 340-354, Noordwijkerhout, The Netherlands, 2002. Springer-Verlag.

[37] Hany Farid. Detecting hidden messages using higher-order statistical models. In Proceedings of the Intl. Conference on Image Processing, volume 2, pages 905-908. IEEE, Jun 2002.

[38] Siwei Lyu. Steganalysis using color wavelet statistics and one-class support vector machines. Master's thesis, Dartmouth College, Hanover, NH, USA, 2002.

[39] Hany Farid. Detecting steganographic messages in digital images. Technical Report TR2001-412, Dartmouth College, Hanover, NH, USA, Mar 2001. 
[40] P. P. Vaidyanathan. Quadrature mirror filter banks, m-band extensions and perfect reconstruction techniques. IEEE Signal Processing Magazine, 4:4-20, Jul 1987.

[41] R. W. Buccigrossi and E. P. Simoncelli. Image compression via joint statistical characterization in the wavelet domain. IEEE Transactions On Image Processing, 8:16881701, 1998.

[42] Ismail Avcibas, Nasir Memon, and Bülent Sankur. Steganalysis using image quality metrics. IEEE Transactions On Image Processing, 12:221-229, Feb 2003.

[43] Ismail Avcibas, Nasir Memon, and Bülent Sankur. Image steganalysis with binary similarity measures. In Proceedings of the Intl. Conference on Image Processing, volume 3, pages 645-648. IEEE, Jun 2002.

[44] Ismail Avcibas, Nasir Memon, and Bülent Sankur. Steganalysis based on image quality metrics. In Proceedings of the Fourth Workshop on Multimedia Signal Processing, pages 517-522. IEEE, Oct 2001.

[45] Ismail Avcibas. Steganalysis using image quality metrics. Master's thesis, Computer and Information Science Polytechnic University, Brooklyn, NY, USA, 2002.

[46] Ueli Maurer. A universal statistical test for random bit generators. Journal of Cryptology, 5:89-105, Feb 1992.

[47] David Freedman, Robert Pisani, and Roger Purves. Statistics. George J. McLeod Limited, Toronto, Canadá, first edition, 1978. ISBN 0-39309-076-0.

[48] The Compuserve Group. Specification of GIF image format, Jul 1990. http: / / www . dcs.ed.ac.uk/home/mxr/gfx/2d/GIF89a.txt.

[49] Philip R. Zimmermann. The Official PGP User's Guide. MIT Press, Boston, MA, USA, 1995. ISBN 0-26274-017-6.

[50] Anderson Rocha, Siome Goldenstein, Heitor A. X. Costa, and Lucas M. Chaves. Esteganografia para proteção e privacidade digital. In $6^{\text {th }}$ SSI, 2004.

[51] Anderson Rocha, Siome Goldenstein, Heitor A. X. Costa, and Lucas M. Chaves. Segurança e privacidade na internet por esteganografia em imagens. In Webmedia \& $L A-W e b$ - Joint Conference 2004, 2004.

[52] Anderson Rocha. Camaleão: um software para segurança digital utilizando esteganografia, 2003. Monografia. Depto. de Ciência da Computação, Universidade Federal de Lavras.

[53] Greg Goth. Steganalysis gets past the hype. IEEE Distributed Systems Online, 6:1-5, 2005. 